\title{
Methylsulfonylmethane enhances BMP-2-induced osteoblast differentiation in mesenchymal stem cells
}

\author{
DON NAM KIM $^{1 *}$, YOUN HEE JOUNG ${ }^{1 *}$, PRAMOD DARVIN $^{1}$, DONG YOUNG KANG ${ }^{1}$, NIPIN SP ${ }^{1}$, \\ HYO JOO BYUN ${ }^{1}$, KWANG HYUN CHO ${ }^{2}$, KYUNG DO PARK ${ }^{3}, \mathrm{HAK} \mathrm{KYO} \mathrm{LEE}^{4}$ and YOUNG MOK YANG ${ }^{1}$ \\ ${ }^{1}$ Department of Pathology, School of Medicine, Institute of Biomedical Science and Technology, \\ Glocal Campus, Konkuk University, Seoul 143-701; ${ }^{2}$ National Institute of Animal Science, RDA, Cheonan 331-801; \\ ${ }^{3}$ Genomics Informatics Center, Hankyong National University, Anseong 456-749; ${ }^{4}$ Department of Animal Biotechnology, \\ Chonbuk National University, Jeonju 561-756, Republic of Korea
}

Received June 19, 2015; Accepted April 25, 2016

DOI: $10.3892 / \mathrm{mmr} .2016 .5274$

\begin{abstract}
As human lifespans have increased, the incidence of osteoporosis has also increased. Methylsulfonylmethane (MSM) affects the process of mesenchymal stem cell (MSC) differentiation into osteoblasts via the Janus kinase 2 (Jak2)/signal transducer and activator of transcription (STAT)5b signaling pathway, and bone morphogenetic protein 2 (BMP-2) is also known to significantly affect bone health. In addition, the phosphorylation of small mothers against decapentaplegic (Smad)1/5/8 regulates the Runt-related transcription factor 2 (Runx2) gene, which encodes a transcription factor for osteoblast differentiation markers. In the present study, the differentiation of MSCs treated with MSM, BMP-2, and their combination were examined. The differentiation of osteoblasts was demonstrated through observation of morphological changes and mineralization, using alizarin red and Von Kossa staining. Western blotting analysis demonstrated that the combination of MSM and BMP-2 increased the phosphorylation of the
\end{abstract}

Correspondence to: Professor Young Mok Yang, Department of Pathology, School of Medicine, Institute of Biomedical Science and Technology, Glocal Campus, Konkuk University, 1 Hwayang-dong, Gwangjin-gu, Seoul 143-701, Republic of Korea

E-mail: ymyang@kku.ac.kr

Professor Hak Kyo Lee, Department of Animal Biotechnology, Chonbuk National University, 567 Baekje-daero, Jeonju 561-756, Republic of Korea

E-mail: breedlee@empal.com

*Contributed equally

Abbreviations: MSM, methylsulfonylmethane; MSC, mesenchymal stem cell; BMP-2, bone morphogenetic protein 2; Runx2, Runt-related transcription factor 2

Key words: methylsulfonylmethane, bone morphogenetic protein-2; small mothers against decapentaplegic $1 / 5 / 8$, osteoblast differentiation, mesenchymal stem cell, mineralization
BMP signaling-associated protein, Smad1/5/8. Combination of MSM and BMP-2 significantly increased osteogenic differentiation and mineralization of the MSCs compared with either MSM or BMP-2 alone. Additionally, reverse transcription-polymerase chain reaction analysis demonstrated that combination of MSM and BMP-2 increased the expression level of the Runx 2 gene and the osteoblast differentiation marker genes, alkaline phosphatase, bone sialoprotein and osteocalcin, in MSCs compared with controls. Thus, the combination of MSM and BMP-2 may promote the differentiation of MSCs into osteoblasts.

\section{Introduction}

Bone is a living organ, which undergoes remodeling throughout life (1). This remodeling involves the removal of mineralized bone by osteoclasts, followed by formation of the bone matrix through osteoblasts, which subsequently becomes mineralized (2). The major regulators of bone remodeling include growth hormone $(\mathrm{GH})$, parathyroid hormone, insulin-like growth factor-1, transforming growth factor (TGF) $\beta$ and bone morphogenetic proteins (BMPs) $(1,3)$. BMP-2, a member of the TGF- $\beta$ superfamily, was originally identified as an inducer of ectopic bone formation (4). The binding of BMP-2 to serine/threonine kinase receptors induces small mothers against decapentaplegic (Smad) phosphorylation, and the phosphorylated Smads then translocate from the cytoplasm to the nucleus to regulate gene transcription (5).

The osteogenic differentiation of mesenchymal stem cells (MSCs) is characterized by the expression of genes, including Runt-related transcription factor 2 (Runx2) and alkaline phosphatase (ALP), followed by extracellular matrix synthesis and mineralization (6). Runx2 is essential in osteoblast differentiation during bone development and remodeling, binding a conserved promoter sequence (R/TACCRCA) and transactivating genes encoding osteogenic proteins, including collagen $\alpha 1$, osteopontin (OPN), bone sialoprotein (BSP) and osteocalcin $(\mathrm{OCN})(7,8)$.

Multiple isotypes of BMP, including BMP-2 and BMP-7, induce MSCs to undergo osteogenic differentiation (9-12). 
BMP-7 and BMP-2 are currently used clinically to induce new bone formation in spine fusion and long bone non-union fractures (13).

Methylsulfonylmethane (MSM) is a simple, volatile organic sulfur, which is non-toxic to humans and contains a compound also known as dimethyl sulfone. Intake of MSM is possible through the consumption of fruits, vegetables, grains and animals (14-17). This compound affects the differentiation of MSCs into osteoblasts by affecting the Janus kinase 2/signal transducer and activator of transcription (STAT)5b signaling pathway $(18,19)$. However, although MSM and BMP-2 affect the bone formation pathway, the effects of the combination of the two on osteoblastic differentiation have received little investigation.

In the present study, the roles of MSM in BMP signaling and the differentiation of MSCs were analyzed. It was hypothesized that MSM enhances BMP-2 signaling and the process of osteoblast differentiation, with Smad1/5/8 and Runx2 as major targets. MSM has potential to be used as a therapeutic agent to treat bone-depleting conditions, such as osteoporosis, where the mineralization of bone is impaired due to decreased osteoblast differentiation.

\section{Materials and methods}

MSC isolation and cell culture. MSCs were isolated from the long bone (femur and tibia) of 12 six-week-old male balb/c mice (Orient Bio, Inc., Seongnam, Korea) following euthanization in a $\mathrm{CO}_{2}$ chamber. The bone marrow was flushed out using needles and $\alpha$-minimal essential medium ( $\alpha$-MEM; Gibco; Thermo Fisher Scientific, Inc., Waltham, MA, USA) without fetal bovine serum (FBS), and unless otherwise stated, the cells were maintained in $\alpha$-MEM containing $10 \% \mathrm{FBS}$ medium at $37^{\circ} \mathrm{C}$ with $5 \% \mathrm{CO}_{2}$ for 7 days. The medium was replaced at 4 day intervals.

Antibodies and reagents. Modified $\alpha$-MEM containing FBS was purchased from Gibco; Thermo Fisher Scientific, Inc. Smad1/5/8 (cat. no. sc-6031), phosphorylated (p)-Smad1/5/8 (cat. no. 9511S) and $\beta$-actin (cat. no. sc-47778) antibodies, and the horseradish peroxidase (HRP)-conjugated rabbit anti-rabbit immunoglobulin G secondary antibodies (cat. no. sc-2004) were purchased from Santa Cruz Biotechnology, Inc. (Santa Cruz, CA, USA) and Cell Signaling Technology, Inc. (Beverly, MA, USA). The anti-actin antibody, MSM, Alizarin red S, ascorbic acid phosphate, $\beta$-glycerophosphate disodium salt hydrate, L-glutamine and 3-(4,5-dimethylthiazol-2-yl)-2,5-diphenyl tetrazolium bromide were obtained from Sigma-Aldrich (St. Louis, MO, USA). Tri-RNA reagent was purchased from Favogen Biotech Corp. (Kaohsiung, Taiwan). The kit used for reverse transcription-polymerase chain reaction (RT-PCR) analysis was obtained from Bioline, Ltd. (London, UK). The ALP, BSP, OCN, Runx 2 and $18 \mathrm{~S}$ primers for use in RT-PCR were purchased from Bioneer (Daejeon, Korea), and enhanced chemiluminescence (ECL) and detection kits were purchased from GE Healthcare (Piscataway, NJ, USA). The Coomassie Protein Assay kit and Restore Western Blot Stripping Buffer were purchased from Pierce Biotechnology, Inc. (Rockford, IL, USA).
Western blot analysis. The MSCs were cultured in the osteogenic medium with MSM (20 mM) and BMP-2 (100 ng/ml) during the initiation of osteoblast differentiation. The cells were lysed in whole lysis buffer, containing $50 \mathrm{mM}$ Tris- $\mathrm{HCl}$ (pH 7.5), 5 mM EDTA, $150 \mathrm{mM} \mathrm{NaCl}, 1 \%$ Triton X-100, and protease and phosphatase inhibitors. Lysates were incubated on ice for $40 \mathrm{~min}$ and then centrifuged at $15,000 \mathrm{x} \mathrm{g}$ for $10 \mathrm{~min}$ at $4^{\circ} \mathrm{C}$. The supernatant was collected and the protein concentrations were determined using a Coomassie protein assay. An equivalent quantity $(20 \mu \mathrm{g})$ of protein extract from each sample was electrophoresed on $10 \%$ sodium dodecyl sulfate polyacrylamide gels and transferred onto nitrocellulose membranes. The membranes were blocked for $1 \mathrm{~h}$ with $5 \%$ bovine serum albumin (Merck Millipore, Darmstadt, Germany) in $20 \mathrm{mM}$ Tris- $\mathrm{HCl}$ (pH 7.6) $137 \mathrm{mM} \mathrm{NaCl}$ and 0.16 Tween 20 (T-TBS), and incubated overnight at $4^{\circ} \mathrm{C}$ with primary antibodies (Smad1/5/8, p-Smad1/5/8 or $\beta$-actin; dilution, 1:1,000). The membranes were then washed three times in T-TBS and incubated with the corresponding anti-rabbit or anti-mouse immunoglobulin G HRP-conjugated secondary antibodies $(1: 2,000)$ in T-TBS with $0.5 \%$ bovine serum albumin for $1 \mathrm{~h}$ under agitation at room temperature. Following incubation, the membranes were washed three times in T-TBS, following which the membranes were developed using the ECL PLUS kit and analyzed using an LAS 4000 machine (Fujifilm, Tokyo, Japan).

$R T-P C R$. The bone marrow MSCs were cultured in osteogenic medium, and the mRNA expression levels were measured following treatment with MSM (20 mM) and BMP-2 (100 ng/ml) at day 5 for ALP, day 14 for BSP and day 21 for OCN. Total RNA was prepared using Tri-RNA reagent, and cDNA was synthesized using the SensiFAST ${ }^{\mathrm{TM}}$ cDNA Synthesis kit (Bioline, Ltd.), according to the manufacturer's protocol. The PCR analysis was performed on aliquots of cDNA to detect Runx2 (301 bp), ALP (454 bp), BSP (81 bp) and OCN (113 bp). The PCR primer sequences were as follows: Runx2, sense 5'-TCACTACCAGCCACCGAGAC-3' and antisense 5'-ACGCCATAGTCCCTCCTTT-3'; ALP, sense 5'-TGG AGCTTCAGAAGCTCAACACCA-3' and antisense 5'-ATC TCGTTGTCTGAGTACCAGTCC-3'; BSP, sense 5'-CAGAGG AGGCAAGAGTCACT-3' and antisense 5'-CTGTCTGGG TGCCAACACTG-3'; OCN, sense 5'-AAGCAGGAGGGC AATAAGGT-3' and antisense 5'-CAAGCAGGGTTAAGC TCACA-3'. For control purposes, the levels of 18S mRNA were measured using the following primer: Sense 5'-CGG CTACCACATCCAAGGAA-3' and antisense 5'-CCGGC G TCCCCTCTTAATC-3'. The PCR reaction was performed as follows: 25 cycles at $94^{\circ} \mathrm{C}$ for $30 \mathrm{sec}, 56^{\circ} \mathrm{C}$ for $30 \mathrm{sec}$ and $72^{\circ} \mathrm{C}$ for $45 \mathrm{sec}$. Following amplification, the PCR products were analyzed on a $1.5 \%$ agarose gel stained with ethidium bromide. mRNA levels were semi-quantified using Multigauge software (version 3.1; Fujifilm Corporation, Tokyo, Japan) with $18 \mathrm{~S}$ as the reference gene.

Mineralization assay. Alizarin Red staining and von Kossa staining of the MSC cultures were performed 21 days following the MSM (20 mM), BMP-2 (100 ng/ml) and combination treatments to evaluate the effects of MSM and BMP-2 on the matrix mineralization of MSCs. The cells were washed 
$\mathbf{A}$

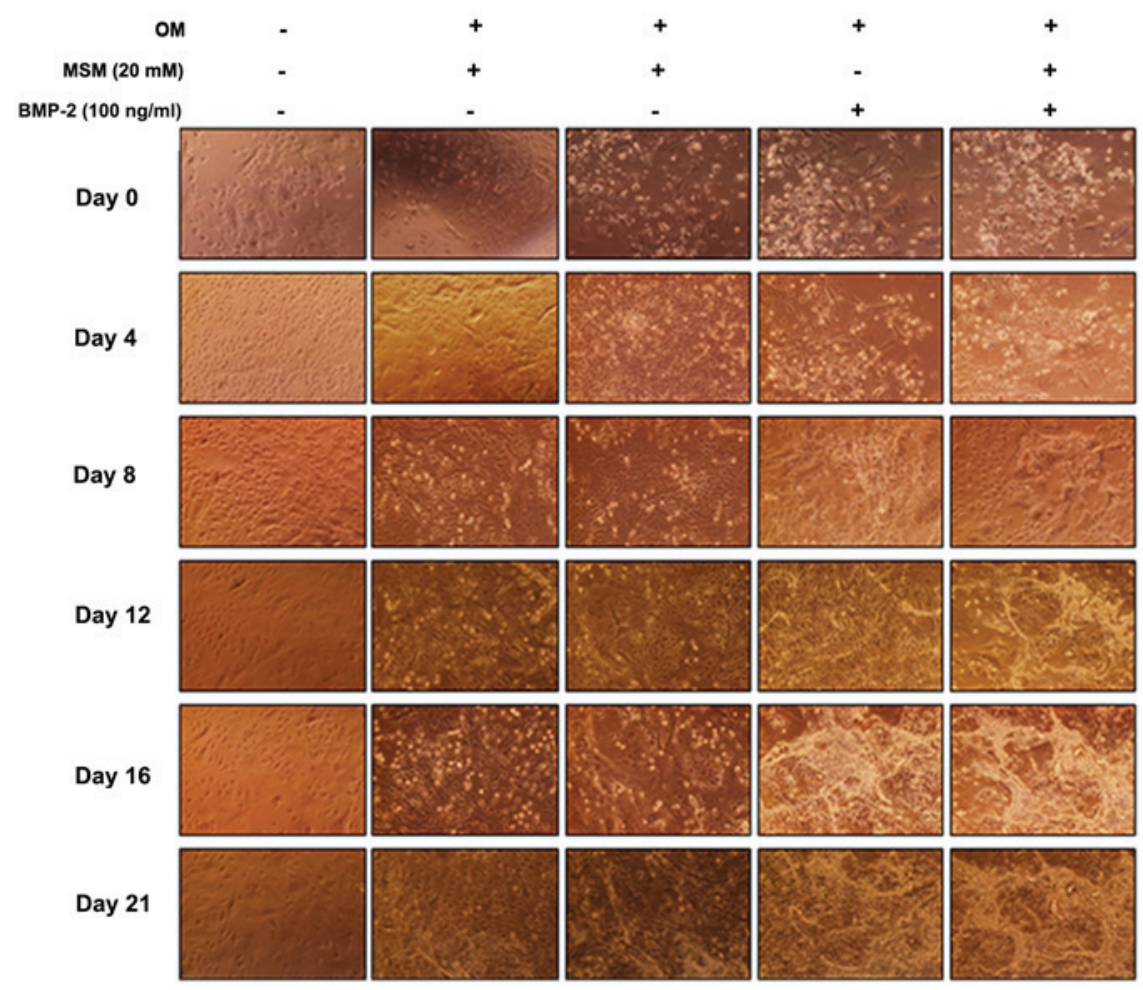

B

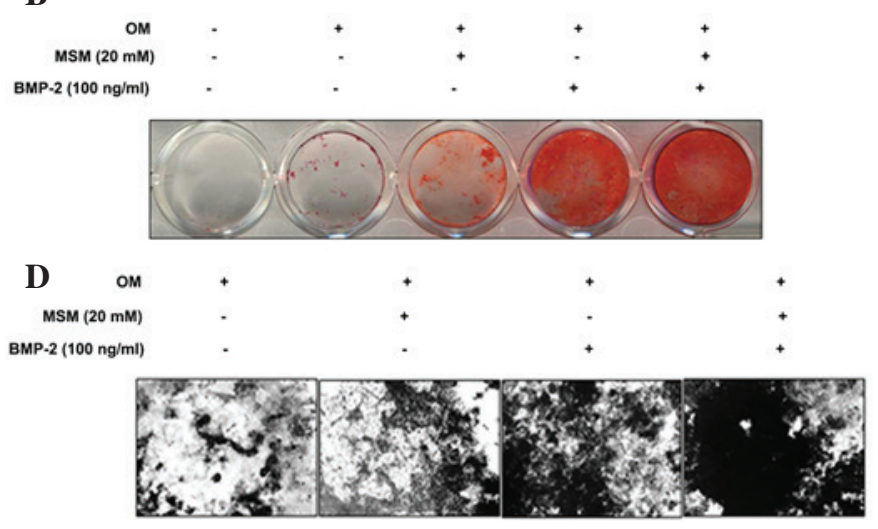

C

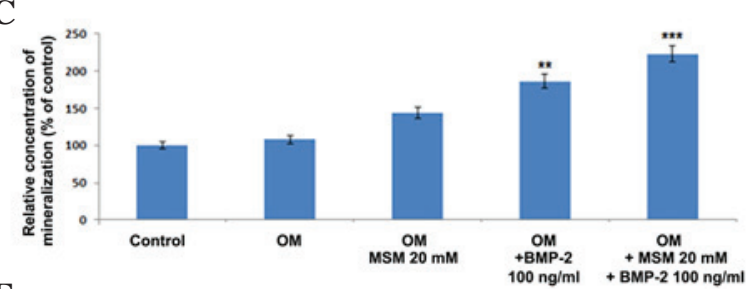

$\mathbf{E}$

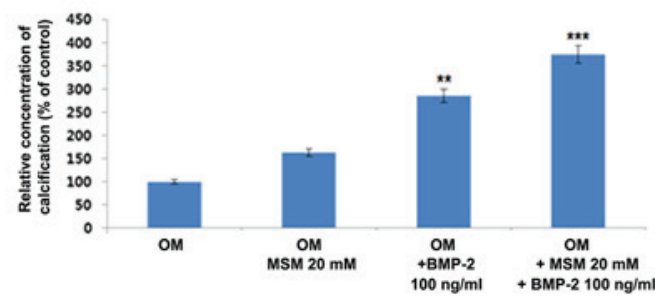

Figure 1. Treatment with a combination of MSM and BMP-2 increases mineralization in MSCs. (A) Analysis of cell morphology following treatment with MSM and BMP-2 for 21 days. The cell morphology was changed from spherical to elongated osteoblast-like structure with organized colonies. Magnification, $\mathrm{x} 400$. (B) Alizarin Red staining showing induced mineralization of MSCs cultured with MSM and BMP-2 for 21 days. (C) Concentration of mineralization relative to the control. (D) Von Kossa staining showing induced calcification of MSCs cultured with MSM and BMP-2 for 21 days. Magnification, x400. (E) Concentration of calcification relative to the control. Data shown are representative of three independent experiments and expressed as the mean \pm standard error of the mean. ${ }^{* *} \mathrm{P}<0.01,{ }^{* * * *} \mathrm{P}<0.001$ vs. OM. OM, osteogenic medium; MSM, methylsulfonylmethane; BMP-2; bone morphogenetic protein-2; MSCs, mesenchymal stem cells.

with phosphate buffered saline (PBS), fixed with $4 \%$ paraformaldehyde in PBS for $15 \mathrm{~min}$, and rinsed with distilled water three times. For Alizarin Red S staining, $40 \mathrm{mM}$ Alizarin Red S ( $\mathrm{pH}$ 4.2) stain was added to the plates and incubated for $10 \mathrm{~min}$ at room temperature; the plates were then rinsed three times with distilled water and washed with PBS to reduce non-specific staining. For the von Kossa staining, the fixed cells were stained with freshly prepared 5\% silver nitrate under ultraviolet light for $1 \mathrm{~h}$ to detect phosphate deposits. Background color was removed using 5\% sodium thiosulfate, and the cells were rinsed three times with distilled water. The cells were observed using phase contrast microscopy (Olympus DP71; Olympus Corporation, Tokyo, Japan), and images were captured using DPC controller software and a Nikon digital camera (Nikon, Tokyo, Japan).

Immunofluorescence microscopy. Immunofluorescence microscopy of the MSC cultures was performed 5 days following the MSM (20 mM), BMP-2 (100 ng/ml), and combination treatments to evaluate the effects of MSM and BMP-2 on Smad1/5/8. The cells were permeabilized with Triton X-100 $(0.1 \%)$ for $15 \mathrm{~min}$ on ice, and blocked with $1 \%$ horse serum (Thermo Fisher Scientific, Inc.) in PBS for $1 \mathrm{~h}$, followed by incubation in a closed humid chamber with the p-Smad1/5/8 antibody in wash buffer ( $1 \%$ horse serum and $0.1 \%$ triton $\mathrm{X}-100$ in PBS), and subsequent incubation with the Alexa 

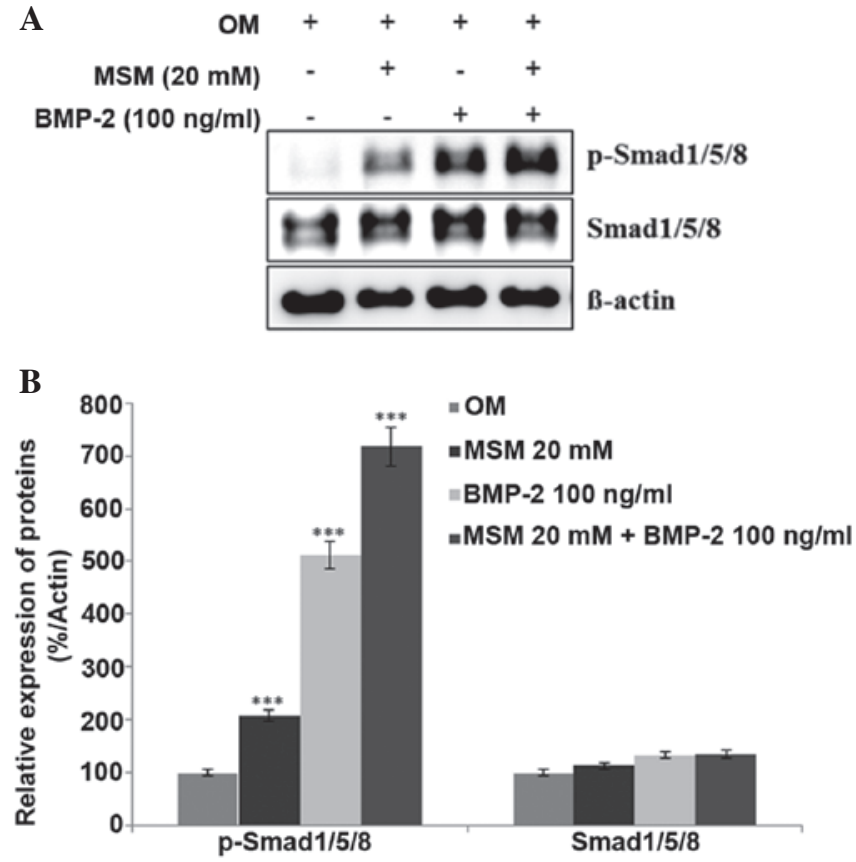

Figure 2. MSM increases BMP-2-induced phosphorylation of Smad1/5/8 in MSCs. (A) MSCs were cultured in OM with MSM and BMP-2 for 21 days. Protein extracts $(20 \mu \mathrm{g})$ were separated on gels, and western blot analysis was performed. $\beta$-actin was used as a protein loading control. (B) Relative protein levels of $\mathrm{p}$-Smad1/5/8 and Smad1/5/8 were determined using densitometric analysis, normalized to the level of $\beta$-actin. Data shown are representative of three independent experiments and expressed as the mean \pm standard error of the mean. One-way analysis of variance was used to detect significant differences $\left({ }^{* * * *} \mathrm{P}<0.001\right.$, vs. OM). Smad $1 / 5 / 8$, small mothers against decapentaplegic 1/5/8; p-Smad1/5/8, phosphorylated-Smad1/5/8; OM, osteogenic medium; MSM, methylsulfonylmethane; BMP-2; bone morphogenetic protein-2; MSCs, mesenchymal stem cells.

Fluor 594 (rabbit) secondary antibody, (Invitrogen; Thermo Fisher Scientific, Inc.) in wash buffer. For nuclear staining, the cells were incubated with TO-PRO3 (Molecular Probes; Thermo Fisher Scientific, Inc.) in wash buffer for $5 \mathrm{~min}$ and rinsed with wash buffer. The slides were then observed under a fluorescence microscope (LSM710; Carl Zeiss, Inc., Oberkochen, Germany).

Statistical analysis. The results of the experiments are expressed as the mean \pm standard error of the mean. Statistical analysis was performed using one-way analysis of variance on the SAS program (version 9.3; SAS Institute, Inc., Cary, NC, USA). $\mathrm{P}<0.05$ was considered to indicate a statistically significant difference.

\section{Results}

Osteogenic differentiation and mineralization are increased in MSCs treated with a combination of MSM and BMP-2. The present study examined the combination effects of MSM and BMP-2 on the differentiation of primary bone marrow MSCs. The cells were cultured with the combination of MSM and BMP-2 for 21 days, following which alterations in morphology were observed (Fig. 1A). Alizarin Red staining was used to visualize the precipitated calcium incorporated into the matrix; it stains intracellular calcium, calcium-binding proteins and proteoglycans, and is thus
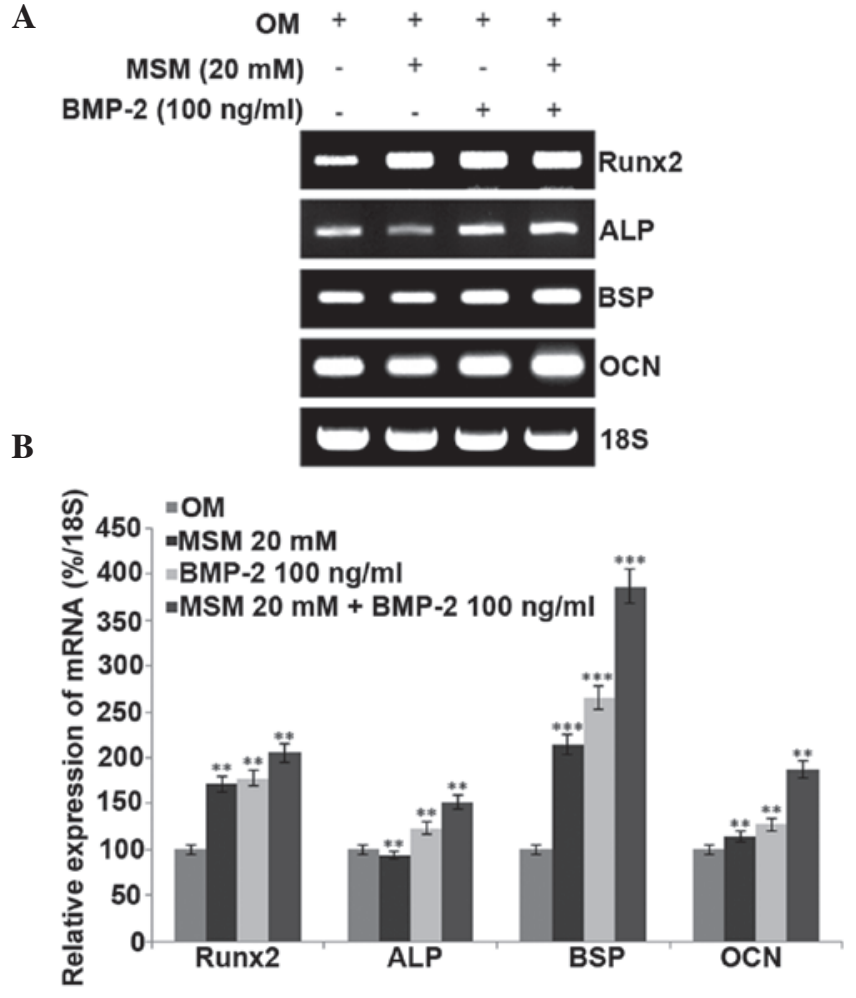

Figure 3. Combined treatment with MSM and BMP-2 enhances the expression of osteoblast differentiation markers in MSCs. (A) Bone marrow MSCs were cultured in OM, and the mRNA expression levels of ALP and Runx2 were detected at day 5, BSP was detected at day 14, and OCN was detected at day 21 following treatment with MSM and BMP-2. Reverse transcription-polymerase chain reaction analysis was performed using primers for ALP, BSP, OCN, Runx 2 and 18S. Total RNA was isolated from the MSCs using an RNeasy kit. 18S was used as a control. (B) mRNA levels of ALP, BSP, OCN and Runx 2 were determined using densitometric analysis and normalized to the level of $18 \mathrm{~S}$. Data shown are representative of three independent experiments and expressed as the mean \pm standard error of the mean. One-way analysis of variance was used to detect significant differences $\left({ }^{* *} \mathrm{P}<0.01\right.$ and ${ }^{* * * *} \mathrm{P}<0.001$, vs. OM). MSCs, mesenchymal stem cells; OM, osteogenic medium; MSM, methylsulfonylmethane; BMP-2; bone morphogenetic protein-2; Runx2, Runt-related transcription factor 2; ALP, alkaline phosphatase; BSP, bone sialoprotein; OCN, osteocalcin.

useful for evaluating the early phases of differentiation. Extracellular calcium deposition by mature osteoblasts was confirmed using von Kossa staining, which detects the phosphate of the calcium phosphate from the secreted matrix. The cells undergoing osteoblast differentiation and mineralization stained positive, enabling comparison in the third week of differentiation. As shown in Fig. 1B, compared with the cells treated with BMP-2 alone, the combination of MSM and BMP-2 increased the area of mineralization, visualized by the Alizarin Red staining for calcium. The relative concentration of mineralization with respect to the control is shown in Fig. 1C. Similar results were verified by the von Kossa staining (Fig. 1D). The percentage of calcification relative to the control is shown in Fig. 1E.

Expression levels of BMP signaling-associated proteins are increased in MSCs treated with a combination of MSM and $B M P-2$. The expression levels of various proteins involved in BMP signaling were assessed using western blot analysis. It was hypothesized that treatment with a combination of MSM 


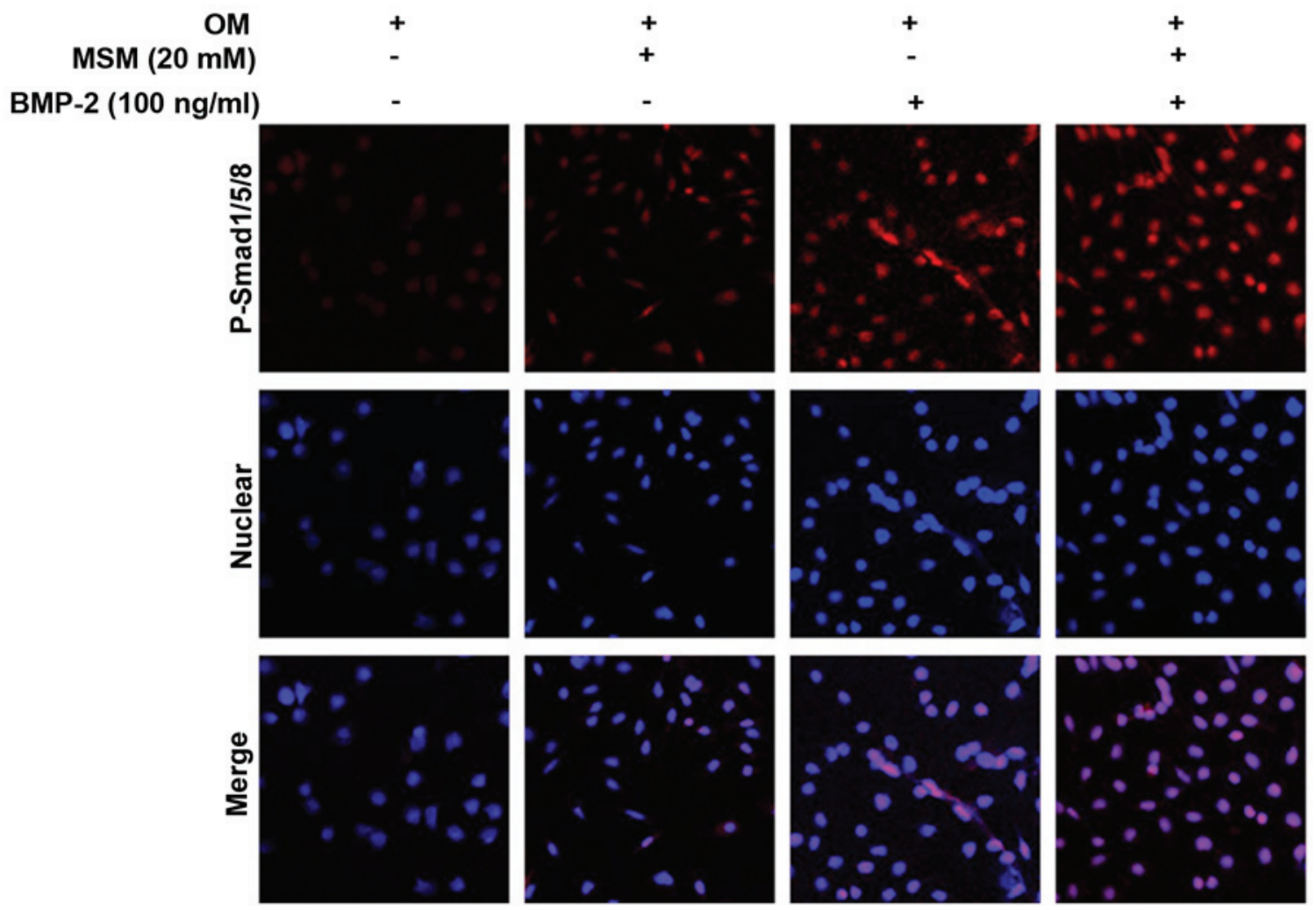

Figure 4. Immunohistochemistry confirmed that MSM increases the expression of BMP-induced p-Smad1/5/8. The cells were treated with primary antibodies specific for $\mathrm{p}-\mathrm{Smad} 1 / 5 / 8$ and detected using Alexa Fluor 594 (rabbit) secondary antibody (magnification, x40). Immunohistochemistry was specific for p-Smad1/5/8. OM, osteogenic medium; MSM, methylsulfonylmethane; BMP, bone morphogenetic protein; p-Smad1/5/8, phosphorylated small mothers against decapentaplegic $1 / 5 / 8$.

and BMP-2 leads to a more marked increase in the expression levels of Smad1/5/8 in MSCs, compared with treatment with BMP-2 alone. As shown in Fig. 2, the combination treatment increased the expression of $\mathrm{p}$-Smad1/5/8 more markedly, compared with the MSCs treated with either BMP-2 or MSM alone. This result confirmed that the combination of MSM and BMP-2 involved the BMP signaling pathways in the MSCs.

mRNA expression levels of Runx2 and osteogenic markers are promoted in MSCs treated with a combination of MSM and $B M P-2$. The present study analyzed the mRNA expression levels of Runx2, ALP, BSP and OCN in the different treatment groups. Runx 2 is a key transcription factor associated with osteoblast differentiation. During the differentiation process, the expression of the early-stage osteogenic differentiation marker, ALP, middle-stage markers, BSP and OPN, and late-stage marker, OCN, increased. However, RT-PCR analysis of the expression of OPN showed no change, compared with the control. The mRNA levels of Runx 2 and osteogenic-specific markers in the MSCs were increased following combined treatment with MSM and BMP-2, compared with the levels following the individual treatments (Fig. 3).

Enhanced complex translocation to the nucleus and changes in the expression of target genes are associated with combined treatment with MSM and BMP-2. Phosphorylation of the Smad1/5/8 protein enhances the expression of osteoblastic marker genes. The present study performed p-Smad1/5/8 localization analysis using immunofluorescence microscopy in the MSCs. The cells were cultured with a combination of MSM and BMP-2 for 5 days, as shown in Fig. 4, and this combination resulted in the upregulation of $\mathrm{p}$-Smad1/5/8 localization from the cytosol to the nucleus.

\section{Discussion}

MSM is a natural organic sulfur from pine tree extract, which is consumed as a 'functional food' with no reported side-effects (15). In a previous study, it was reported that STAT5b is involved in the MSM-induced osteoblastic differentiation of MSCs and is essential in the MSM-induced GH signaling of $\mathrm{C} 3 \mathrm{H} 10 \mathrm{~T} 1 / 2$ cells (18). In addition, it has been found that Hwanggeumchal sorghum extract induces the expression of BMP-7 in osteoblastic cells (3). BMP-2 is important in bone formation, bone regeneration, fracture healing and osteophyte formation (20-23); thus, the present study hypothesized that the combination of MSM and BMP-2 affects the gene expression of osteoblast markers and osteoblastic differentiation.

In the present study, the effect of MSM on BMP-2 activity in the differentiation of MSCs into osteoblasts was investigated. Among BMPs, BMP-2 is involved in bone mineralization, an essential step in the late stage of osteoblast differentiation (24). BMP-2 signals by binding with BMP receptor type II and phosphorylates BMP receptor type I. This phosphorylation activates Smad1/5/8, an important 
protein for the activation of downstream proteins responsible for differentiation.

In the present study, an increase in the phosphorylation of Smad1/5/8 was observed when MSM and BMP-2 were used as a combination treatment. This combination increased the expression of $\mathrm{p}$-Smad1/5/8 in MSCs more markedly, compared with either BMP-2 or MSM alone, indicting that the ability of MSM to enhance the effects of BMP-2 in osteoblast differentiation.

Interleukin-6R can trigger the phosphorylation of Smad4, which leads to formation of a heterocomplex with Smad1/5/8 and translocation to the nucleus. In the nucleus, this Smad complex binds with DNA and other transcription factors, including Dlx5 and Runx2, promoting their activity $(25,26)$. This binding activates the transcription and translation of multiple proteins involved in the differentiation of MSCs into osteoblasts. Runx2 regulates its target genes, including OPN, BSP and OCN, by binding and transactivating the promoter region (27). The role of Runx 2 in regulating target genes is not always positive, and negative regulation by Runx 2 has also been reported (27).

To confirm the ability of MSM to enhance BMP-2-mediated osteoblast differentiation, the present study analyzed multiple molecular markers. The combination treatment resulted in transcriptional upregulation of the receptor molecules involved in BMP-2 signaling. Additionally, ALP, BSP and OCN were markedly increased following the combination treatment, compared with the effects of BMP-2 or MSM alone. These elevated expression levels of the early-stage, middle-stage and late-stage marker genes, ALP, BSP and OCN, respectively, suggested that MSM enhanced the effects of BMP-2 in MSCs.

Osteoblast differentiation is estimated by the rate of calcium mineralization in MSCs. The role of BMP-2 in bone mineralization has been previously reported, and the present study confirmed the ability of MSM to enhance BMP-2 activity. This ability was further confirmed in the present study using calcification assays. Bone mineralization/ calcification is the process mediated by osteoblastic cells, and the level of calcium deposition can be used to detect the level of osteoblastic cells differentiation from MSCs (28). In the present study, the rates of mineralization of the MSCs following treatment with BMP-2, MSM, and the two in combination were examined using the Alizarin Red and von Kossa staining assays. The results showed a high rate of mineralization in the combination-treated group, compared with either BMP-2 or MSM alone. Morphological analysis of the MSCs at 21 days also showed calcium deposition, which indicated differentiation. The calcium mineralization was suggested the ability of MSM to synergize with BMP-2 to induce osteoblast differentiation in the MSCs. The analysis of $\mathrm{p}$-Smad1/5/8 using confocal microscopy confirmed the osteoblast differentiation.

In conclusion, the present study demonstrated that the combination of MSM and BMP-2 can be used to stimulate BMP-2 signaling, thereby promoting osteoblast differentiation. MSM positively regulated BMP-2-induced osteoblastic differentiation via Smad1/5/8, suggesting that MSM has a significant role in bone formation. Thus, the results of the current study that MSM may potentially be useful as a candidate therapeutic agent for bone-depleting conditions, such as osteoporosis, were osteoblast differentiation and bone mineralization are impaired. Further investigations are required to elucidate the implications of MSM-induced BMP-2 signaling using in vivo models of bone resorption mechanisms.

\section{Acknowledgements}

This study was supported by the Basic Science Research Program through the National Research Foundation of Korea funded by the Ministry of Science, ICT \& Future Planning (grant no. 2013R1A1A3013276) and was partially supported by the Next Generation BioGreen 21 Program RDA, Republic of Korea (grant no. PJ001104401).

\section{References}

1. Hadjidakis DJ and Androulakis II: Bone remodeling. Ann NY Acad Sci 1092: 385-396, 2006.

2. Zuo C, Huang Y, Bajis R, Sahih M, Li YP, Dai K and Zhang X: Osteoblastogenesis regulation signals in bone remodeling. Osteoporos Int 23: 1653-1663, 2012.

3. Joung YH, Lim EJ, Darvin P, Jang JW, Park KD, Lee HK, Kim HS, Cho BW, Park T, Chung S, et al: Hwanggeumchal sorghum extract enhances BMP7 and GH signaling through the activation of Jak2/STAT5B in MC3T3-E1 osteoblastic cells. Mol Med Rep 8: 891-896, 2013.

4. Wozney JM: The potential role of bone morphogenetic proteins in periodontal reconstruction. J Periodontol 66: 506-510, 1995.

5. Chen D, Zhao M and Mundy GR: Bone morphogenetic proteins. Growth Factors 22: 233-241, 2004.

6. Dieudonne FX, Sévère N, Biosse-Duplan M, Weng JJ, Su Y and Marie PJ: Promotion of osteoblast differentiation in mesenchymal cells through Cbl-mediated control of STAT5 activity. Stem Cells 31: 1340-1349, 2013.

7. Schroeder TM, Jensen ED and Westendorf JJ: Runx2: A master organizer of gene transcription in developing and maturing osteoblasts. Birth Defects Res C Embryo Today 75: 213-225, 2005.

8. Long F: Building strong bones: Molecular regulation of the osteoblast lineage. Nat Rev Mol Cell Biol 13: 27-38, 2011.

9. Katagiri T, Yamaguchi A, Ikeda T, Yoshiki S, Wozney JM, Rosen V, Wang EA, Tanaka H, Omura S and Suda T: The non-osteogenic mouse pluripotent cell line, C3H10T1/2, is induced to differentiate into osteoblastic cells by recombinant human bone morphogenetic protein-2. Biochem Biophys Res Commun 172: 295-299, 1990.

10. Wang EA, Israel DI, Kelly S and Luxenberg DP: Bone morphogenetic protein-2 causes commitment and differentiation in C3H10T1/2 and 3T3 cells. Growth Factors 9: 57-71, 1993.

11. Puleo DA: Dependence of mesenchymal cell responses on duration of exposure to bone morphogenetic protein-2 in vitro. J Cell Physiol 173: 93-101, 1997.

12. Ducy P, Zhang R, Geoffroy V, Ridall AL and Karsenty G: Osf2/Cbfa1: A transcriptional activator of osteoblast differentiation. Cell 89: 747-754, 1997.

13. Gautschi OP, Frey SP and Zellweger R: Bone morphogenetic proteins in clinical applications. ANZ J Surg 77: 626-631, 2007.

14. Morton JI and Siegel BV: Effects of oral dimethyl sulfoxide and dimethyl sulfone on murine autoimmune lymphoproliferative disease. Proc Soc Exp Biol Med 183: 227-230, 1986.

15. Horváth K, Noker PE, Somfai-Relle S, Glávits R, Financsek I and Schauss AG: Toxicity of methylsulfonylmethane in rats. Food Chem Toxicol 40: 1459-1462, 2002.

16. Kim YH, Kim DH, Lim H, Baek DY, Shin HK and Kim JK: The anti-inflammatory effects of methylsulfonylmethane on lipopolysaccharide-induced inflammatory responses in murine macrophages. Biol Pharm Bull 32: 651-656, 2009.

17. Caron JM, Bannon M, Rosshirt L, Luis J, Monteagudo L, Caron JM and Sternstein GM: Methyl sulfone induces loss of metastatic properties and reemergence of normal phenotypes in a metastatic cloudman S-91 (M3) murine melanoma cell line. PLoS One 5: e11788, 2010. 
18. Joung YH, Lim EJ, Darvin P, Chung SC, Jang JW, Do Park K Lee HK, Kim HS, Park T and Yang YM: MSM enhances GH signaling via the Jak2/STAT5b pathway in osteoblast-like cells and osteoblast differentiation through the activation of STAT5b in MSCs. PLoS One 7: e47477, 2012.

19. Darvin P, Joung YH and Yang YM: JAK2-STAT5B pathway and osteoblast differentiation. JAKSTAT 2: e24931, 2013.

20. Takaoka K, Nakahara H, Yoshikawa H, Masuhara K, Tsuda T and Ono K: Ectopic bone induction on and in porous hydroxyapatite combined with collagen and bone morphogenetic protein. Clin Orthop Relat Res: 250-254, 1988.

21. Nakase T, Nomura S, Yoshikawa H, Hashimoto J, Hirota S, Kitamura Y, Oikawa S, Ono K and Takaoka K: Transient and localized expression of bone morphogenetic protein 4 messenger RNA during fracture healing. J. Bone Miner Res 9: 651-659, 1994.

22. Nakase T, Miyaji T, Tomita T, Kaneko M, Kuriyama K, Myoui A, Sugamoto K, Ochi T and Yoshikawa H: Localization of bone morphogenetic protein-2 in human osteoarthritic cartilage and osteophyte. Osteoarthritis Cartilage 11: 278-284, 2003.

23. Kaneko K, Higuchi C, Kunugiza Y, Yoshida K, Sakai T, Yoshikawa $\mathrm{H}$ and Nakata K: Hyaluronan inhibits BMP-induced osteoblast differentiation. FEBS Lett 589: 447-454, 2015.
24. Prall WC, Haasters F, Heggebö J, Polzer H, Schwarz C, Gassner C, Grote S, Anz D, Jäger M, Mutschler W and Schieker M: Mesenchymal stem cells from osteoporotic patients feature impaired signal transduction but sustained osteoinduction in response to BMP-2 stimulation. Biochem Biophys Res Commun 440: 617-622, 2013.

25. Shea CM, Edgar CM, Einhorn TA and Gerstenfeld LC: BMP treatment of C3H10T1/2 mesenchymal stem cells induces both chondrogenesis and osteogenesis. J Cell Biochem 90: 1112-1127, 2013.

26. Kim HJ, Park JW, Lee KH, Yoon H, Shin DH, Ju UI, Seok SH, Lim SH, Lee ZH, Kim HH and Chun YS: Plant homeodomain finger protein 2 promotes bone formation by demethylating and activating Runx2 for osteoblast differentiation. Cell Res 24: 1231-1249, 2014.

27. Westendorf JJ, Zaidi SK, Cascino JE, Kahler R, van Wijnen AJ, Lian JB, Yoshida M, Stein GS and Li X: Runx2 (Cbfa1, AML-3) interacts with histone deacetylase 6 and represses the p21 (CIP1/WAF1) promoter. Mol Cell Biol 22: 7982-7992, 2002 .

28. Mbalaviele G, Sheikh S, Stains JP, Salazar VS, Cheng SL, Chen D and Civitelli R: Beta-catenin and BMP-2 synergize to promote osteoblast differentiation and new bone formation. J Cell Biochem 94: 403-418, 2005. 